\title{
A MODIFIED RATIO-PRODUCT ESTIMATOR OF POPULATION MEAN IN THE PRESENCE OF MEDIAN AND COEFFICIENT OF VARIATION OF THE AUXILIARY VARIABLE IN STRATIFIED RANDOM SAMPLING
}

\author{
Abubakar Yahaya and Umar Kabir Abdullahi \\ Department of Statistics, Ahmadu Bello University, Zaria, Nigeria. \\ abubakaryahaya@abu.edu.ng, +2348069334466. \\ umarkabir9@gmail.com, +2348109554170.
}

\begin{abstract}
For the past decades, the estimation of population mean is one of the challenging aspects in sampling survey techniques and much effort has been employed to improve the precision of estimates. In this research work, we proposed a modified ratio-product estimator of population mean of the variable of interest using median and coefficient of variation of the auxiliary variable in stratified random sampling scheme. The expression of bias and MSE of the proposed estimator have been obtained under large sample approximation, asymptotically optimum estimator (AOE) is identified with its approximate MSE formula. Estimator based on "estimated optimum values" was also investigated. Theoretical and empirical comparison of proposed estimator with some other ratio and product estimator justified the performance of the proposed estimator. There is a minimum of 15 percent reduction in the MSE from each of the existing ratio and product estimators considered. Thus most preferred over the existing estimators for the use in practical application.

Keywords: bias, mean square error, auxiliary variable, optimum estimator, stratified random sampling, study variable.
\end{abstract}

\section{INTRODUCTION}

Auxiliary variable(s) has been widely discussed in sampling theory and population study. Auxiliary variables are in used in survey sampling to obtain improved sampling designs and to achieve more precision in the estimates of some population parameters such as the mean and the variance of the variable of interest. This information may be used at both the design stage, execution stage and estimation stage of designing a survey.

The estimation of population mean is a burning issue in sampling theory and many efforts have been made to improve the precision of the estimate. In survey sample literature, a great variety of techniques for using auxiliary information by means of ratio, regression and product methods have been used in the presence of one or more auxiliary variables. It is also established that if the regression line of the variable under study and the auxiliary variable is through the origin and are positively correlated the best estimator to be used is ratio estimator likewise if the regression line of the variable under study and the auxiliary variable is through the origin and are negatively correlated the best estimator to be used is product estimator, on the other hand when the regression line does not pass through the origin but makes an intercept along the $y$-axis and there is weak correlation either positive or negative between the auxiliary variable and the variable of interest the best to used is the linear regression estimator (Okafor, 2002).

In modern surveys, the scientific technique for selecting a sample is that of selecting a probability sample that is usually based on a stratification of the population. It is well known that stratification is one of the design tools that gives increased in precision. In stratified design the population under investigation is divided into different strata so as to obtain the homogeneity with each stratum and sample observation are drawn within each stratum by well known simple random sampling.

The disadvantage of using simple random sampling (SRS) when the population is not homogeneous have been comprehensively documented in the well known literature (see for instance, (Cochran 1977), more so, research by several authors reveal that ratio product estimator performs better than ratio and product type estimators in simple random sampling (SRS) under stratification and other certain conditions. These therefore motivate us to propose an estimator in stratified random sampling design and study its properties.

\section{Background of the Study}

Consider a finite population $P=\left(P_{1}, P_{2}, \ldots, P_{N}\right)$ be of $N$ divided into $L$ homogeneous strata of size $N_{h}(h=1,2,3, \ldots L)$. A sample of size $n_{h}$ is drawn from each stratum using simple random sampling without replacement (SRSWOR). Let $y$ be the study variate taking values $y_{h i}$ ( $i^{\text {th }}$ observation from $h^{\text {th }}$ stratum) and let $x$ be the auxiliary variate taking the values $x_{h i}$. 
Bajopas Volume 10 Number 1 June, 2017

Moreover, let $\bar{y}_{s t}=\sum_{h=1}^{L} W_{h} \bar{y}_{h}, \bar{x}_{s t}=\sum_{h=1}^{L} W_{h} \bar{x}_{h}$ be the unbiased estimators of the population mean $\bar{Y}$ (the study variate) and $\bar{X}$ (the auxiliary variate, respectively).

Where,

$W_{h}=\frac{N_{h}}{N}$, is the weight of $h^{\text {th }}$ stratum,

$\bar{y}_{h}=\frac{1}{n_{h}} \sum_{h=1}^{n_{h}} y_{h i}$, is the mean of the study variate $y$ in $h^{\text {th }}$ stratum,

$\bar{x}_{h}=\frac{1}{n_{h}} \sum_{h=1}^{n_{h}} x_{h i}$, is the mean of the auxiliary variate $x$ in $h^{\text {th }}$ stratum,

Remarks:

1 To ensure the applicability of the estimator, we assume the population values of the study variate are known in the entire stratum. This is a reasonable assumption as survey samplers usually obtain such information inexpensively through pilot survey or past experience.

2 A ratio-product estimate of population mean $\bar{Y}$ can be made in two ways. One is to make a separate ratio estimate of the total of each stratum and add their totals. An alternative estimate is to derive a single combined ratio.

To have a survey estimate of the population mean $\bar{Y}$ of the study variable $Y$, assuming the knowledge of the population mean $\bar{X}_{h}$ of the $h^{\text {th }}$ stratum $(h=1,2,3, \ldots, L)$ of the auxiliary variable $X$, we define a separate ratio estimator as

$\bar{y}_{R s t 1}=\sum_{h=1}^{L} W_{h} \widehat{R}_{h} \bar{X}_{h}$,

Where $\hat{R}_{h}=\bar{y}_{h} / \bar{x}_{h}, \bar{x}_{h} \neq 0$ is the estimate of ratio $R_{h}=\bar{Y}_{h} / \bar{X}_{h}, \bar{X}_{h} \neq 0$, of the $h^{\text {th }}$ - stratum in the population. The estimator is only efficient if the variables are strongly positively correlated.

The separate product estimator

$\bar{y}_{P s t 1}=\sum_{h=1}^{L} W_{h} \frac{\hat{P}_{h}}{\bar{X}_{h}}$

Where $\hat{P}_{h}=\bar{y}_{h} \bar{x}_{h}$ is the estimate of product $P_{h}=\bar{Y}_{h} \bar{X}_{h}, \bar{X}_{h} \neq 0$ of the $h^{\text {th }}-$ stratum in the population. The estimator is only efficient if the variables are strongly negatively correlated.

To the first order of approximation (i.e, to terms of order $0\left(n_{h}^{-1}\right)$ ), the variance of equation (2.1) and (2.2) above are respectively given by

$$
\begin{aligned}
& \operatorname{MSE}\left(\bar{y}_{R s t 1}\right)=\sum_{h=1}^{L} W_{h}^{2} \frac{\left(1-f_{h}\right)}{n_{h}} \bar{Y}_{h}^{2}\left[C_{h y}^{2}+C_{h x}^{2}\left\{1-2 K_{h}\right\}\right] \\
& \operatorname{MSE}\left(\bar{y}_{P s t 1}\right)=\sum_{h=1}^{L} W_{h}^{2} \frac{\left(1-f_{h}\right)}{n_{h}} \bar{Y}_{h}^{2}\left[C_{h y}^{2}+C_{h x}^{2}\left\{1+2 K_{h}\right\}\right]
\end{aligned}
$$

where

$$
\begin{aligned}
& f_{h}=\frac{n_{h}}{N_{h}}, K_{h}=\rho_{h} \frac{C_{h y}}{C_{h x}}, \rho_{h}=\frac{S_{h x y}}{S_{h x} S_{h Y}}, C_{h y}=\frac{S_{h y}}{\bar{Y}_{h}}, C_{h x}=\frac{h_{h x}}{X_{h}} \\
& S_{h x y}=\frac{\sum_{i=1}^{N_{h}}\left(x_{h i}-\bar{X}_{h}\right)\left(y_{h i}-\bar{Y}_{h}\right)}{\left(N_{h}-1\right)}, S_{h x}^{2}=\frac{\sum_{i=1}^{N_{h}}\left(x_{h i}-\bar{X}_{h}\right)^{2}}{\left(N_{h}-1\right)}, S_{h y}^{2}=\frac{\sum_{i=1}^{N_{h}}\left(y_{h i}-\bar{Y}_{h}\right)^{2}}{\left(N_{h}-1\right)} .
\end{aligned}
$$


Bajopas Volume 10 Number 1 June, 2017

The direct generalization of Sisodia and Dwivedi (1981) transformation of the auxiliary variable in stratified random sampling design by Kadilar and Cingi (2003), is defined as

$\bar{y}_{s t . S D R}=\bar{y}_{s t} \frac{\sum_{h=1}^{L}\left(\bar{X}_{h}+C_{x h}\right)}{\sum_{h=1}^{L}\left(\bar{x}_{h}+C_{x h}\right)}$
$\bar{y}_{s t . S D P}=\bar{y}_{s t} \frac{\sum_{h=1}^{L}\left(\bar{x}_{h}+C_{x h}\right)}{\sum_{h=1}^{L}\left(\bar{X}_{h}+C_{x h}\right)}$

They defined $\bar{X}_{S D}=\sum_{h=1}^{L}\left(\bar{X}_{h}+C_{x h}\right), \bar{x}_{S D}=\sum_{h=1}^{L}\left(\bar{x}_{h}+C_{x h}\right)$. Then equation (2.5) and (2.6) will be

$\bar{y}_{s t . S D}=\bar{y}_{s t} \frac{\bar{X}_{S D}}{\bar{x}_{S D}}=\widehat{R}_{S D} \bar{X}_{S D}$

Where $\widehat{R}_{S D}=\bar{y}_{s t} \frac{\bar{y}_{s t}}{\bar{x}_{S D}}$. It should be noted that the difference between combined ratio and Sisodia and Dwivedi (1981) estimator is only $\widehat{R}_{S D}$. Thus, bias and MSE of those estimators can be given in the same way like equations (2.8) and (2.9), equation (2.10) and (2.11) respectively as $\operatorname{Bias}\left(\bar{y}_{s t . S D R}\right)=\frac{1}{\bar{X}_{S D}}\left[\sum_{h=1}^{K} W_{h}^{2} \gamma_{h}\left(R_{S D} S_{x h}^{2}-S_{y x h}\right)\right]$

$\operatorname{MSE}\left(\bar{y}_{s t . S D R}\right)=\sum_{h=1}^{K} W_{h}^{2} \gamma_{h}\left(S_{y h}^{2}-2 R_{S D} S_{y x h}+R_{S D}^{2} S_{x h}^{2}\right)$

$\operatorname{Bias}\left(\bar{y}_{s t . S D P}\right)=\frac{1}{\bar{X}_{S D}}\left[\sum_{h=1}^{K} W_{h} \gamma_{h}\left(R_{S D} S_{x h}^{2}+2 S_{y x h}\right)\right]$

$\operatorname{MSE}\left(\bar{y}_{s t . S D R}\right)=\sum_{h=1}^{K} W_{h}^{2} \gamma_{h}\left(S_{y h}^{2}+2 R_{S D} S_{y x h}-R_{S D}^{2} S_{x h}^{2}\right)$

$R_{S D R}=R_{S D P}=\frac{\bar{Y}_{s t}}{\bar{X}_{S D}}=\frac{\sum_{h=1}^{K} W_{h} \bar{X}_{h}}{\sum_{h=1}^{K}\left(\bar{X}_{h}+C_{x h}\right)}$

The Subramani and Kumarapandiyan (2012) stratified ratio and product estimator are define in equation (2.13) and (2.14) respectively

$\bar{y}_{R s t 2}=\bar{y}_{s t} \frac{\sum_{h=1}^{K}\left(\bar{X}_{h} C_{x h}+M_{d}\right)}{\sum_{h=1}^{K}\left(\bar{x}_{h} C_{x h}+M_{d}\right)}$

$\bar{y}_{\text {Pst } 2}=\bar{y}_{s t} \frac{\sum_{h=1}^{K}\left(\bar{x}_{h} C_{x h}+M_{d}\right)}{\sum_{h=1}^{K}\left(\bar{X}_{h} C_{x h}+M_{d}\right)}$

The corresponding bias and MSE of the estimator in equation (2.13) and (2.14) are given in equation (2.15) and (2.16) respectively

$$
\begin{aligned}
& \operatorname{Bias}\left(\bar{y}_{R s t 2}\right)=\frac{1}{\bar{X}_{R s t 2}}\left[\sum_{h=1}^{K} W_{h}^{2} \gamma_{h}\left(R_{R s t 2} S_{x h}^{2}-S_{y x h}\right)\right] \\
& \operatorname{MSE}\left(\bar{y}_{R s t 2}\right)=\sum_{h=1}^{K} W_{h}^{2} \gamma_{h}\left(S_{y h}^{2}-2 R_{R s t 2} S_{y x h}+R_{R s t 2}^{2} S_{x h}^{2}\right)
\end{aligned}
$$




$$
\begin{aligned}
& \operatorname{Bias}\left(\bar{y}_{P_{s t 2}}\right)=\frac{1}{\bar{X}_{P_{s t 2}}}\left[\sum_{h=1}^{K} W_{h}^{2} \gamma_{h}\left(R_{P_{s t 2}} S_{x h}^{2}+S_{y x h}\right)\right] \\
& \operatorname{MSE}\left(\bar{y}_{P_{s t 2}}\right)=\sum_{h=1}^{K} W_{h}^{2} \gamma_{h}\left(S_{y h}^{2}-R_{P_{s t 2}}^{2} S_{x h}^{2}+2 R_{P_{s t 2}} S_{y x h}\right) \\
& R_{R s t 2}=R_{P_{s t 2}}=\frac{\sum_{h=1}^{K} W_{h} \bar{X}_{h} C_{x h}}{\sum_{h=1}^{K} W_{h} \bar{X}_{h} C_{x h}+M_{d}}
\end{aligned}
$$

\section{Proposed Estimator}

Motivated by the direct generalization of Sisodia and Dwivedi (1981), Housila and Neha-Agnihotri (2008), Singh and Vishwakarma (2011), Subramani and Kumarapandiyan (2012),

Using the various definitions above we proposed the separate and combine ratio-product estimator respectively as

$$
\begin{aligned}
& T_{R P}^{(s)}=\sum_{h=1}^{L} W_{h} \bar{y}_{h}\left\{\delta_{h} \frac{\sum_{h=1}^{L} W_{h} \bar{X}_{h} C_{x h}+M_{d h}}{\sum_{h=1}^{L} W_{h} \bar{x}_{h} C_{x h}+M_{d h}}+\left(1-\delta_{h}\right) \frac{\sum_{h=1}^{L} W_{h} \bar{x}_{h} C_{x h}+M_{d h}}{\sum_{h=1}^{L} W_{h} \bar{X}_{h} C_{x h}+M_{d h}}\right\} \\
& T_{R P}^{(C)}=\bar{y}_{s t}\left\{\delta_{s t} \frac{\sum_{h=1}^{L}\left(\bar{X}_{s t} C_{x s t}+M_{d s t}\right)}{\sum_{h=1}^{L}\left(\bar{x}_{s t} C_{x s t}+M_{d s t}\right)}+\left(1-\delta_{s t}\right) \frac{\sum_{h=1}^{L}\left(\bar{x}_{s t} C_{x s t}+M_{d s t}\right)}{\sum_{h=1}^{L}\left(\bar{X}_{s t} C_{x s t}+M_{d s t}\right)}\right\}
\end{aligned}
$$

Where $\bar{y}_{h}=\frac{1}{n_{h}} \sum_{h=i}^{n_{h}} y_{h i}$ and $\bar{x}_{h}=\frac{1}{n_{h}} \sum_{h=i}^{n_{h}} x_{h i}$ are unbiased estimators of the population means $\left(\bar{Y}_{h}, \bar{X}_{h}\right)$ respectively, ' $C_{x h}$ ' and ' $M_{d h}$ ' are the known characteristics positive scalars of the auxiliary variable $X$ respectively and $\delta_{s t}$ is a real constant to be determined such that the mean square error of $T_{R P}^{(S)}$ is minimum. The family of estimators $T_{R P}^{(S)}$ reduces to the following set of known estimators,

(i) For $\left(C_{x h}, M_{d h}, \delta_{h}\right)=\left(0,1, \delta_{h}\right), T_{R P}^{(S)} \rightarrow \bar{y}_{s t}$ (usual unbiased stratified estimator)

(ii) For $\left(C_{x h}, M_{d h}, \delta_{h}\right)=\left(0,1, \delta_{h}\right), T_{R P}^{(S)} \rightarrow Q_{1}=\left[\delta_{s t} \bar{y}_{s t}\left(\frac{\sum_{h=1}^{L} W_{h} \bar{X}_{h}}{\sum_{h=1}^{L} W_{h} \bar{x}_{h}}\right)+\left(1-\delta_{s t}\right) \bar{y}_{s t}\left(\frac{\sum_{h=1}^{L} W_{h} \bar{x}_{h}}{\sum_{h=1}^{L} W_{h} \bar{X}_{h}}\right)\right]$

which is due to Singh and Espejo (2003)

$$
\begin{aligned}
& \text { (iii) For }\left(C_{x h}, M_{d h}, \delta_{h}\right)=\left(0,1, \delta_{h}\right), \\
& T_{R P}^{(S)} \rightarrow Q_{2}\left[\delta_{h} \bar{y}_{s t}\left(\frac{\sum_{h=1}^{L} W_{h}\left(\bar{X}_{h}+C_{x h}\right)}{\sum_{h=1}^{L} W_{h}\left(\bar{x}_{h}+C_{x h}\right)}\right)+\left(1-\delta_{h}\right) \bar{y}_{s t}\left(\frac{\sum_{h=1}^{L} W_{h}\left(\bar{x}_{h}+C_{x h}\right)}{\sum_{h=1}^{L} W_{h}\left(\bar{X}_{h}+C_{x h}\right)}\right)\right]
\end{aligned}
$$

envisaged by Singh and Tailor (2005), where $C_{x h}$ is the known population coefficient of variation and $M_{d h}$ is the median of the auxiliary $X$ respectively, many other ratio-product estimators can be generated from $T_{R P}^{(S)}$ by putting any suitable parameters rather than values of $\left(C_{x h}, M_{d h}, \delta_{h}\right)$.

\section{bias and Mean Square Error (MSE)}

To obtain the bias and mean square error (MSE) of the proposed family of estimator $T_{R P}^{(S)}$ in $(3.71)$, we write

Let $e_{0 h}=\frac{\left(\bar{y}_{h}-\bar{Y}_{h}\right)}{\bar{Y}_{h}}$ and $e_{1 h}=\frac{\left(\bar{x}_{h}-\bar{X}_{h}\right)}{\bar{X}_{h}}$

Then $\bar{y}_{h}=\bar{Y}_{h}\left(1+e_{0 h}\right)$ and $\bar{x}_{h}=\bar{X}_{h}\left(1+e_{1 h}\right)$ 
Bajopas Volume 10 Number 1 June, 2017

Such that $E\left(e_{0 h}\right)=E\left(e_{1 h}\right)=0$

$E\left(e_{0 h}^{2}\right)=\frac{1-f_{h}}{n_{h}} C_{y h}^{2}, E\left(e_{1 h}^{2}\right)=\frac{1-f_{h}}{n_{h}} C_{x h}^{2}, E\left(e_{0 h} e_{1 h}\right)=\frac{1-f_{h}}{n_{h}} K_{h} C_{x h}^{2}$

where $W_{h}=\frac{N_{h}}{N} f_{h}=\frac{n_{h}}{N_{h}}, K_{h}=\rho \frac{C_{y}}{C_{x}}, \rho_{h}=\frac{S_{x y h}}{S_{x h} S_{Y h}}, C_{y h}=\frac{S_{y h}}{\bar{Y}_{h}}, C_{x h}=\frac{S_{x h}}{\bar{X}_{h}}$.

$S_{x y h}=\frac{\sum_{h=1}^{L}\left(x_{h i}-\bar{X}_{h}\right)\left(y_{h i}-\bar{Y}_{h}\right)}{\left(N_{h}-1\right)}, S_{x h}^{2}=\frac{\sum_{h=1}^{L}\left(x_{h i}-\bar{X}_{h}\right)^{2}}{\left(N_{h}-1\right)}, S_{y h}^{2}=\frac{\sum_{h=1}^{L}\left(y_{h i}-\bar{Y}_{h}\right)^{2}}{\left(N_{h}-1\right)}$.

And expanding (3.71) in terms of $e_{h}{ }^{\prime} s$, we have

$T_{R P}^{(S)}=\bar{Y}_{h}\left(1+e_{0 h}\right)\left[\delta_{h}\left(1+\theta_{h} e_{1 h}\right)^{-1}+\left(1-\delta_{h}\right)\left(1+\theta_{h} e_{1 h}\right)\right]$

Where $\theta_{h}=\frac{\bar{X}_{h} C_{x h}}{\left(\bar{X}_{h} C_{x h}+M_{d h}\right)}$

We assume that $\left|\theta_{h} e_{1 h}\right|<1$, so that the expression $\left(1+\theta_{h} e_{1 h}\right)^{-1}$ can be expanded to a convergent infinite

$$
\begin{aligned}
& \text { series using binomial theorem. Hence from (3.3) we have. } \\
& T_{R P}^{(S)}=\bar{Y}_{s t}\left(1+e_{0 h}\right)\left[\delta_{h}\left(1-\theta_{h} e_{1 h}+\theta_{h}^{2} e_{1 h}^{2}-\theta_{h}^{3} e_{1 h}^{3}+\theta_{h}^{4} e_{1 h}^{4} \cdots\right)+\left(1-\delta_{h}\right)\left(1+\theta_{h} e_{1 h}\right)\right] \\
& =\bar{Y}_{s t}\left[\delta_{h}\left(1+e_{0 h}\right)\left(1-\theta_{h} e_{1 h}+\theta_{h}^{2} e_{1 h}^{2}-\theta_{h}^{3} e_{1 h}^{3}+\theta_{h}^{4} e_{1 h}^{4} \ldots\right)+\left(1-\delta_{h}\right)\left(1+e_{0 h}\right)\left(1+\theta_{h} e_{1 h}\right)\right] \\
& =\bar{Y}_{s t}\left[\begin{array}{l}
\left.\delta_{h}\left(1+e_{0 h}-\theta_{h} e_{1 h}+\theta_{h}^{2} e_{1 h}^{2}-\theta_{h} e_{1 h} e_{0 h}+\theta_{h}^{2} e_{1 h} e_{0 h}+\theta_{h}^{2} e_{1 h}^{2} e_{0 h}-\theta_{h}^{3} e_{1 h}^{3}+\theta_{h}^{4} e_{1 h}^{4}-\theta^{3} e_{1 h}^{3} e_{0 h}+\ldots\right)+\right] \\
\left(1-\delta_{h}\right)\left(1+e_{0 h}+\theta_{h} e_{1 h}+\theta_{h} e_{1 h} e_{0 h}\right)
\end{array}\right] \\
& =\bar{Y}_{s t}\left[1+e_{0 h}+\theta_{h} e_{1 h}+\theta_{h} e_{1 h} e_{0 h}+\delta_{h}\left(\begin{array}{l}
\left.1+e_{0 h}-\theta e_{1 h}-\theta_{h} e_{1 h} e_{0 h}+\theta_{h}^{2} e_{1 h}^{2}+\theta_{h}^{2} e_{1 h}^{2} e_{0 h}-\theta_{h}^{3} e_{1 h}^{3}+\theta_{h}^{4} e_{1 h}^{4}-\theta_{h}^{3} e_{1 h}^{3} e_{0 h}+\right) \\
\ldots-1-e_{0 h}-\theta_{h} e_{1 h}-\theta_{h} e_{0 h} e_{1 h}
\end{array}\right)\right] \\
& =\bar{Y}_{s t}\left[1+e_{0 h}+\theta_{h} e_{1 h}+\theta_{h} e_{1 h} e_{0 h}+\delta_{h}\left(-2 \theta_{h} e_{1 h} e_{0 h}-2 \theta_{h} e_{0 h} e_{1 h}+\theta_{h}^{2} e_{1 h}^{2}+\theta_{h}^{2} e_{1 h}^{2} e_{0 h}-\theta_{h}^{3} e_{1 h}^{3}+\theta_{h}^{4} e_{1 h}^{4}-\theta_{h}^{3} e_{1 h}^{3} e_{0 h}+\ldots\right)\right] \\
& =\bar{Y}_{s t}\left[1+e_{0 h}-\left(1-2 \delta_{h}\right) \theta_{h} e_{1 h}+\left(1-2 \delta_{h}\right) \theta_{h} e_{1 h} e_{0 h}+\delta_{h} \theta_{h}^{2} e_{1 h}^{2}+\delta_{h}\left(\theta_{h}^{2} e_{1 h}^{2} e_{0 h}-\theta_{h}^{3} e_{1 h}^{3}+\theta_{h}^{4} e_{1 h}^{4}-\theta_{h}^{3} e_{1 h}^{3} e_{0 h}+\ldots\right)\right]
\end{aligned}
$$

We assume that the contribution of terms involving powers in $e_{0 h}$ and $e_{1 h}$ higher than the second is negligible, being of order $1 / n^{v}$, where $v>1$. Thus, from the above expression we write to a first order of approximation, $T_{R P}^{(S)} \cong \bar{Y}_{s t}\left[1+e_{0 h}-\left(1-2 \delta_{h}\right) \theta_{h} e_{1 h}+\left(1-2 \delta_{h}\right) \theta_{h} e_{1 h} e_{0 h}+\delta_{h} \theta_{h}^{2} e_{1 h}^{2}+\delta_{h} \theta_{h}^{2} e_{1 h}^{2} e_{0 h}\right]$, or $\left(T_{R P}^{(S)}-\bar{Y}_{s t}\right)=\bar{Y}_{s t}\left[e_{0 h}-\left(1-2 \delta_{h}\right) \theta_{h} e_{1 h}+\left(1-2 \delta_{h}\right) \theta_{h} e_{1 h} e_{0 h}+\delta \theta_{h}^{2} e_{1 h}^{2}+\delta_{h} \theta_{h}^{2} e_{1 h}^{2} e_{0 h}\right]$,

Taking the expectation of both side of (3.4), we obtained the bias of $\left(T_{R P}^{(S)}\right)$ to the first degree of approximation as

$\operatorname{Bias}\left(T_{R P}^{(S)}\right)=\sum_{h=1}^{L} \frac{\left(1-f_{h}\right)}{n_{h}} \theta_{h} \bar{Y}_{h}\left[\mathrm{~K}_{h}+\delta_{h}\left(\theta_{h}-2 K_{h}\right)\right] C_{x h}^{2}$

Equation (3.5) will vanishes if $\delta_{h}=\frac{K_{h}}{\left(2 \mathrm{~K}_{h}-\theta_{h}\right)}$

Thus for $\delta_{h}=\frac{K_{h}}{\left(2 \mathrm{~K}_{h}-\theta_{h}\right)} T_{R P}^{(S)}$ is almost unbiased. 
Bajopas Volume 10 Number 1 June, 2017

Squaring both side of the equation (3.4), and neglecting the terms of $e_{h}{ }^{\prime} s$ having power greater than two we have

$\left(T_{R P}^{(S)}-\bar{Y}_{h}\right)^{2}=\bar{Y}_{h}^{2}\left[e_{0 h}^{2}+\left(1-2 \delta_{h}\right) \theta_{h}\left\{\left(1-2 \delta_{h}\right) \theta_{h} e_{1 h}^{2}+2 e_{1 h} e_{0 h}\right\}\right]$

Taking the expectation of both sides of (3.6), we get the mean square error MSE of $T_{R P}^{(S)}$ to the first order of approximation as

$\operatorname{MSE}\left(T_{R P}^{(S)}\right)=\sum_{h=1}^{L} \frac{\left(1-f_{h}\right)}{n_{h}} \bar{Y}_{h}^{h}\left[C_{y h}^{2}+\theta_{h}\left(1-2 \delta_{h}\right) C_{x h}^{2}\left\{\left(1-2 \delta_{h}\right) \theta_{h}+2 K_{h}\right\}\right]$

To obtain the value of $\delta_{h}$ that minimizes the $M S E$ of $\left(T_{R P}^{(S)}\right)$, we take the partial derivative of the $M S E$ of $\left(T_{R P}^{(S)}\right)$ with respect to $\delta_{h}$ and equate it to zero.

$\delta_{h}=\frac{1}{2}\left(1+\frac{K_{h}}{\theta_{h}}\right)=\delta_{0 h}$ (Optimum Value)

Putting (3.8) in (3.7), we get the Asymptotically Optimum Estimator (AOE) as

$T_{R P O}^{(S)}=\sum_{h=1}^{L} \frac{\bar{y}_{h}}{2}\left[\left(1+\frac{K_{h}}{\theta_{h}}\right)\left(\frac{\bar{X}_{h} C_{x h}+M_{d h}}{\bar{x}_{h} C_{x h}+M_{d h}}\right)+\left(1-\frac{K_{h}}{\theta_{h}}\right)\left(\frac{\bar{x}_{h} C_{x h}+M_{d h}}{\bar{X}_{h} C_{x h}+M_{d h}}\right)\right]$

Substitution of (3.8) in (3.7) yield the minimum $M S E$ of $\left(T_{R P}^{(S)}\right)$ or the $M S E$ of asymptotically optimum estimator $(A O E)\left(T_{R P}^{(S)}\right)$ as

$M S E_{\text {min }}\left(T_{R P}^{(S)}\right)=\sum_{h=1}^{L} \frac{\left(1-f_{h}\right)}{n_{h}} S_{y h}^{2}\left(1-\rho_{h}^{2}\right)=\operatorname{MSE}\left(T_{R P O}^{(S)}\right)$

which is equal to the approximate $M S E$ of stratified regression estimator as

$\bar{y}_{l r S T}=\bar{y}_{s t}+\sum_{h=1}^{L} \hat{\beta}_{h}\left(\bar{X}_{h}-\bar{x}_{h}\right)$

Where $\hat{\beta}_{h}=s_{x y h} / s_{x h}^{2}$, is the sample estimate of the population regression coefficient $\beta_{h}$ of $y_{h}$ on $x_{h}$

$s_{x y h}=\frac{\sum_{h=1}^{L}\left(x_{h i}-\bar{x}_{h}\right)\left(y_{h i}-\bar{y}_{h}\right)}{\left(n_{h}-1\right)}, s_{x h}^{2}=\frac{\sum_{h=1}^{L}\left(x_{h i}-\bar{x}_{h}\right)^{2}}{\left(n_{h}-1\right)}$.

It is to be noted the $A O E$ of $\left(T_{R P O}^{(S)}\right)$ in (3.9) depends on $K_{h}$ and $\theta_{h}$, so the $A O E$ of $\left(T_{R P}^{(S)}\right)$ can be used in practice only when $K_{h}$ and $\theta_{h}$ are known. Here it should be mention that $\theta_{h}$ is a function of known quantities $\left(C_{x h}, M_{d h}, \delta_{h}\right)$. So only the value of $K_{h}$ should be known for making the use of $A O E$ of $\left(T_{R P O}^{(S)}\right)$ in practice. The value of $K_{h}$ can be made known quite accurately either from pilot study or past data or experience gathered in due course of time. This problem has been discussed among others by Murthy (1967), Reddy (1978), Srivankataramana and Tracy (1980). Thus, the value of $K_{h}$ can be guessed quite accurately and such an estimator can be used in practice.

\section{Allowable Departure}

Let $k_{0 h}$ be an estimate or guessed value of $K_{h}$ with

$k_{0 h}=K_{h}\left(1+\eta_{h}\right)$, then

$\delta_{h}=\frac{1}{2}\left(1+\frac{K_{h}}{\theta_{h}}\right)=\frac{1}{2}\left(1+\frac{K_{h}}{\theta_{h}}+\frac{1}{\theta_{h}}\left(k_{0 h}-K_{h}\right)\right)=\delta_{0 h}+\frac{\eta_{h} K_{h}}{2 \theta_{h}}$

Putting (4.1) in (3.7) we obtain the MSE of $\left(T_{R P}^{(S)}\right)$ as

$\operatorname{MSE}\left(T_{R P}^{(S)}\right)=\left(T_{R P O}^{(S)}\right)+\left(\frac{1-f_{h}}{n_{h}}\right) \rho_{h}^{2} \eta_{h}^{2} S_{y h}^{2}$ 
Bajopas Volume 10 Number 1 June, 2017

$$
\begin{aligned}
& \Rightarrow \operatorname{MSE}\left(T_{R P}^{(S)}\right)-\operatorname{MSE}\left(T_{R P O}^{(S)}\right)=\left(\frac{1-f_{h}}{n_{h}}\right) \rho_{h}^{2} \eta_{h}^{2} S_{y h}^{2} \\
& \Rightarrow \frac{M S E\left(T_{R P}^{(S)}\right)-M S E\left(T_{R P O}^{(S)}\right)}{M S E\left(T_{R P O}^{(S)}\right)}=\left(\frac{\rho_{h}^{2} \eta_{h}^{2}}{\left(1-\rho_{h}^{2}\right)}\right)
\end{aligned}
$$

It follows from (4.2) that the proportional increase in $M S E$ of $\left(T_{R P}^{(S)}\right)$ over that of $A O E$ of $\left(T_{R P O}^{(S)}\right)$ is less than $\gamma_{h}$ if,

$$
\begin{gathered}
\frac{\rho_{h}^{2} \eta_{h}^{2}}{\left(1-\rho_{h}^{2}\right)}<\gamma_{h} \\
\text { i.e. }\left|\eta_{h}\right|<\sqrt{\frac{\left(1-\rho_{h}^{2}\right)}{\rho_{h}^{2}} \gamma_{h}},
\end{gathered}
$$

Which clearly shows that to ensure only a small relative increase in $M S E$ of $\left(T_{R P}^{(s)}\right),\left|\eta_{h}\right|$ must be in the neighborhood of "zero" if $\rho$ is high but can depart substantially from "zero" if $\rho_{h}$ is moderate.

\section{Efficiency Comparison}

It is well known under SRSWOR that

$\operatorname{Var}\left(\bar{y}_{s t}\right)=\sum_{h=1}^{L} W_{h}^{2}\left(\frac{1-f_{h}}{n_{h}}\right) S_{y h}^{2}=\sum_{h=1}^{L} W_{h}^{2} \bar{Y}_{h}^{2}\left(\frac{1-f_{h}}{n_{h}}\right) S_{y h}^{2}$

From (3.7) and (5.1) we have

$\operatorname{Var}\left(\bar{y}_{s t}\right)-\operatorname{MSE}\left(T_{R P}^{(S)}\right)=\sum_{h=1}^{L} W_{h}^{2} \bar{Y}^{2} \frac{\left(1-f_{h}\right)}{n_{h}} \theta_{h} C_{x h}^{2}\left(2 \delta_{h}-1\right)\left[\left(1-2 \delta_{h}\right) \theta_{h}+2 K_{h}\right]$

which is non- negative if

$$
\min \left\{\frac{1}{2}, \frac{1}{2}\left\{1+\frac{2 \sum_{h=1}^{L} K_{h}}{\sum_{h=1}^{L} \theta_{h}}\right)\right\}<\delta_{s t}<\max \left\{\frac{1}{2}, \frac{1}{2}\left(1+\frac{2 \sum_{h=1}^{L} K_{h}}{\sum_{h=1}^{L} \theta_{h}}\right)\right\}
$$

It is to be noted that for $\delta_{h}=1$, the estimator $T_{R P}^{(S)}$ reduces to the stratified ratio-type estimator $\left(T_{R s t 2}\right)=\bar{y}_{s t}\left(\frac{\sum_{h=1}^{L} W_{h} \bar{X}_{h} C_{x h}+M_{d h}}{\sum_{h=1}^{L} W_{h} \bar{x}_{h} C_{x h}+M_{d h}}\right)$

where for $\delta_{h}=0$, the estimator $T_{R P}^{(S)}$ turns out to be the stratified product-type estimator

$\left(T_{P_{s t 2}}\right)=\bar{y}_{s t}\left(\frac{\sum_{h=1}^{L} W_{h} \bar{x}_{h} C_{x h}+M_{d h}}{\sum_{h=1}^{L} W_{h} \bar{X}_{h} C_{x h}+M_{d h}}\right)$

To the first degree of approximation the mean squared errors of $T_{R s t 2}, T_{P s t 2}$ are respectively given by

$$
\begin{aligned}
& \operatorname{MSE}\left(T_{R s t 2}\right)=\sum_{h=1}^{L} W_{h}^{2} \bar{Y}_{h}^{2} \frac{\left(1-f_{h}\right)}{n_{h}}\left[C_{y h}^{2}+\theta_{h} C_{x h}^{2}\left\{\theta_{h}-2 K_{h}\right\}\right] \\
& \operatorname{MSE}\left(T_{P_{s t 2}}\right)=\sum_{h=1}^{L} W_{h}^{2} \bar{Y}_{h}^{2} \frac{\left(1-f_{h}\right)}{n_{h}}\left[C_{y h}^{2}+\theta_{h} C_{x h}^{2}\left\{\theta_{h}+2 K_{h}\right\}\right]
\end{aligned}
$$

From (3.7), (5.5) and (5.6), we have

$$
\begin{aligned}
& \operatorname{MSE}\left(T_{R s t 2}\right)-\operatorname{MSE}\left(T_{R P}^{(S)}\right)=\sum_{h=1}^{L}\left(\frac{4\left(1-f_{h}\right)}{n_{h}}\right) \theta_{h} W_{h}^{2} \bar{Y}_{h}^{2} C_{x h}^{2}\left[\left(1-\delta_{h}\right)\left(\delta_{h} \theta_{h}-K_{h}\right)\right] \\
& \operatorname{MSE}\left(T_{P s t 2}\right)-\operatorname{MSE}\left(T_{R P}^{(S)}\right)=\sum_{h=1}^{L}\left(\frac{4\left(1-f_{h}\right)}{n_{h}}\right) \theta_{h} W_{h}^{2} \bar{Y}_{h}^{2} C_{x h}^{2}\left[\left(1-\delta_{h}\right)\left(\delta_{h} \theta_{h}+K_{h}\right)\right]
\end{aligned}
$$

It follows from (5.7) and (5.8) that the ratio-product estimator $T_{R P}^{(S)}$ is more efficient than

(i) The ratio type estimator $T_{R s t 2}$ if 
Bajopas Volume 10 Number 1 June, 2017

$\min \left(\frac{\sum_{h=1}^{L} K_{h}}{\sum_{h=1}^{L} \theta_{h}}, 1\right)<\delta_{s t}<\max \left(\frac{\sum_{h=1}^{L} K_{h}}{\sum_{h=1}^{L} \theta_{h}}, 1\right)$

(ii) The product- type estimator $T_{P 2}$ if

$\min \left(\left(1+\frac{\sum_{h=1}^{L} K_{h}}{\sum_{h=1}^{L} \theta_{h}}\right), 0\right)<\delta_{s t}<\max \left(\left(1+\frac{\sum_{h=1}^{L} K_{h}}{\sum_{h=1}^{L} \theta_{h}}\right), 0\right)$

Further, if we set $\left(C_{x h}, M_{d h}\right)=(1,0)$ in (5.3) and (5.4) the ratio-type estimator $T_{R s t 2}$ and product-type $T_{P s t 2}$ estimators respectively reduces to

$T_{R s t 2} \rightarrow T_{R s t 2}=\bar{y}_{s t} \frac{\bar{X}_{s t}}{\bar{x}_{s t}}$ (usual stratified ratio estimator)

$T_{R s t 2} \rightarrow T_{R s t 2}=\bar{y}_{s t} \frac{\bar{X}_{s t}}{\bar{x}_{s t}}$ (usual stratified product estimator)

Putting $\left(C_{x h}, M_{d h}\right)=(1,0)$ in (5.2) and (5.3) we get the mean squared errors of usual stratified ratio and product estimators respectively as

$\operatorname{MSE}\left(T_{R s t 1}\right)=\sum_{h=1}^{L} W_{h}^{2} \bar{Y}_{h}^{2} \frac{\left(1-f_{h}\right)}{n_{h}}\left[C_{y h}^{2}+C_{x h}^{2}\left\{1-2 K_{h}\right\}\right]$

$\operatorname{MSE}\left(T_{R s t 1}\right)=\sum_{h=1}^{L} W_{h}^{2} \bar{Y}_{h}^{2} \frac{\left(1-f_{h}\right)}{n_{h}}\left[C_{y h}^{2}+C_{x h}^{2}\left\{1+2 K_{h}\right\}\right]$

From (3.7), (5.13) and (5.14) we have

$\operatorname{MSE}\left(T_{R s t 1}\right)-\operatorname{MSE}\left(T_{R P}^{(S)}\right)=\sum_{h=1}^{L} W_{h}^{2} \bar{Y}_{h}^{2} \frac{\left(1-f_{h}\right)}{n_{h}}\left(1+\theta_{h}-2 \delta_{h} \theta_{h}\right)\left(1-\theta_{h}-2 K_{h}+2 \theta_{h} \delta_{h}\right) C_{x h}^{2}$

$\operatorname{MSE}\left(T_{P s t 1}\right)-\operatorname{MSE}\left(T_{R P}^{(S)}\right)=\sum_{h=1}^{L} W_{h}^{2} \bar{Y}_{h}^{2} \frac{\left(1-f_{h}\right)}{n_{h}}\left(1-\theta_{h}+2 \delta_{h} \theta_{h}\right)\left(1+\theta_{h}+2 K_{h}-2 \theta_{h} \delta_{h}\right) C_{x h}^{2}$

From (5.15) and (5.16), we note that the ratio-product estimator $T_{R P}^{(S)}$ is better than

(i) The stratified ratio type estimator $T_{R s t}$ if

$\min \left\{\left(\frac{1+\sum_{h=1}^{L} W_{h} \theta_{h}}{2 \sum_{h=1}^{L} W_{h} \theta_{h}}\right),\left(\frac{\sum_{h=1}^{L}\left(W_{h} 2 K_{h}+\theta_{h} / 2\right)-1}{2 \sum_{h=1}^{L} W_{h} \theta_{h}}\right)\right\}<\delta_{s t}<\max \left\{\left\{\left(\frac{1+\sum_{h=1}^{L} W_{h} \theta_{h}}{2 \sum_{h=1}^{L} W_{h} \theta_{h}}\right),\left(\frac{\sum_{h=1}^{L}\left(W_{h} 2 K_{h}+\theta_{h} / 2\right)-1}{2 \sum_{h=1}^{L} W_{h} \theta_{h}}\right)\right\}\right\}$

(ii) The product- type estimator $T_{P s t 1}$ if

$\min \left\{\left(\frac{\sum_{h=1}^{L} W_{h} \theta_{h}-1}{2 \sum_{h=1}^{L} W_{h} \theta_{h}}\right),\left(\frac{\sum_{h=1}^{L}\left(W_{h} 2 K_{h}+\theta_{h} / 2\right)+1}{2 \sum_{h=1}^{L} W_{h} \theta_{h}}\right)\right\}<\delta_{s t}<\max \left\{\left\{\left(\frac{\sum_{h=1}^{L} W_{h} \theta_{h}-1}{2 \sum_{h=1}^{L} W_{h} \theta_{h}}\right),\left(\frac{\sum_{h=1}^{L}\left(W_{h} 2 K_{h}+\theta_{h} / 2\right)+1}{2 \sum_{h=1}^{L} W_{h} \theta_{h}}\right)\right\}\right\}$

Estimator based on the Optimum value

The optimum value of $\delta_{h}$ at (3.8) is

$\delta_{0 h}=\frac{1}{2}\left(1+\frac{K_{h}}{\theta_{h}}\right)$

Where $\theta_{h}$ is a known quantity and

$K_{h}=\rho_{h} \frac{C_{y h}}{C_{x h}}=\frac{\rho_{h} S_{y h} \bar{X}_{h}}{S_{x h} \bar{Y}_{h}}=\frac{S_{x y h}}{R S_{x h}^{2}}=\frac{\beta_{h}}{R_{h}}$

Replacing $\beta_{h}$ and $R_{h}$ by their consistent estimators

$\hat{\beta}_{h}=\frac{s_{x y h}}{s_{x h}^{2}}$ and $\hat{R}_{h}=\frac{\bar{y}_{h}}{\bar{x}_{h}}$ respectively.

From (6.1) we get a consistent estimator of $\delta_{0 h}$ as

$\delta_{0 h}=\frac{1}{2}\left(1+\frac{\hat{K}_{h}}{\theta_{h}}\right)$ 
Bajopas Volume 10 Number 1 June, 2017

where $\hat{K}_{h}=\frac{\hat{\beta}_{h}}{R_{h}}$

If the experimenter is unable to guess the value of $K_{h}$, then it is worth advisable to replace $K$ by $\widehat{K}_{h}$ in (3.9). Thus, the estimator based on the estimated 'optimum' value.

$\widehat{T}_{R P O}^{(S)}=\frac{\bar{y}_{s t}}{2}\left[\left(1+\frac{\hat{K}_{h}}{\theta_{h}}\right)\left(\frac{\sum_{h=1}^{L} W_{h}\left(\bar{X}_{h} C_{x h}+M_{d h}\right)}{\sum_{h=1}^{L} W_{h}\left(\bar{x}_{h} C_{x h}+M_{d h}\right)}\right)+\left(1-\frac{\widehat{K}_{h}}{\theta_{h}}\right)\left(\frac{\sum_{h=1}^{L} W_{h}\left(\bar{x}_{h} C_{x h}+M_{d h}\right)}{\sum_{h=1}^{L} W_{h}\left(\bar{X}_{h} C_{x h}+M_{d h}\right)}\right)\right]$

To obtain the MSE of $\widehat{T}_{R P O}^{(S)}$ we write

Let $e_{2 h}=\frac{\left(\hat{k}_{h}-K_{h}\right)}{K_{h}}$, then $\hat{k}_{h}=K_{h}\left(1+e_{2 h}\right)$

with $E\left(e_{2 h}\right)=K_{h}+o\left(n^{-1}\right)$, expanding (6.3) in terms of $e_{h}$ 's we have

$\widehat{T}_{R P O}^{(S)}=\frac{\bar{Y}_{s t}}{2}\left(1+e_{0 h}\right)\left[\left(1+\frac{K_{h}}{\theta_{h}}\left(1+e_{2 h}\right)\right)\left(1+\theta_{h} e_{1 h}\right)^{-1}+\left(1-\frac{K_{h}}{\theta_{h}}\left(1+e_{2 h}\right)\right)\left(1+\theta_{h} e_{1 h}\right)\right]$

From (6.4) we have

$=\frac{\bar{Y}_{s t}}{2}\left(1+e_{o h}\right)\left[\left\{1+\frac{K_{h}}{\theta_{h}}\left(1+e_{2 h}\right)\right\}\left(1-\theta_{h} e_{1 h}+\theta_{h}^{2} e_{1 h}-\theta_{h}^{3} e_{1 h}+\theta_{h}^{4} e_{1 h}+\ldots\right)+\left\{1-\frac{K_{h}}{\theta_{h}}\left(1+e_{2 h}\right)\right\}\left(1-\theta_{h} e_{1 h}\right)\right]$
$=\frac{\bar{Y}_{s t}}{2}\left(1+e_{o h}\right)\left[\begin{array}{l}\left.1+\frac{K_{h}}{\theta_{h}}\left(1+e_{2 h}\right)-\theta_{h} e_{1 h}-K_{h}\left(e_{1 h}+e_{1 h} e_{2 h}\right)+K_{h} \theta_{h}\left(e_{1 h}^{2}+e_{2 h} e_{1 h}^{2}\right)+\ldots+1-\frac{K_{h}}{\theta_{h}}\left(1+e_{2 h}\right)+\theta_{h} e_{1 h}\right] \\ -K_{h}\left(e_{1 h}+e_{1 h} e_{2 h}\right)\end{array}\right.$
$=\frac{\bar{Y}_{s t}}{2}\left(1+e_{o h}\right)\left[2-2 K_{h}\left(e_{1 h}+e_{1 h} e_{2 h}\right)+K_{h} \theta_{h}\left(e_{1 h}^{2}+e_{2 h} e_{1 h}^{2}\right)+\ldots\right]$
$=\bar{Y}_{s t}\left(1+e_{o h}\right)\left[1-K_{h}\left(e_{1 h}+e_{1 h} e_{2 h}\right)+\frac{K_{h} \theta_{h}}{2}\left(e_{1 h}^{2}+e_{2 h} e_{1 h}^{2}\right)+\ldots\right]$
$=\bar{Y}_{s t}\left[1+e_{o h}-K_{h}\left(e_{1 h}+e_{0 h} e_{1 h}+e_{1 h} e_{2 h}+e_{0 h} e_{1 h} e_{2 h}\right)+\frac{K_{h} \theta_{h}}{2}\left(e_{1 h}^{2}+e_{2 h} e_{1 h}^{2}+e_{0 h} e_{1 h}^{2}+e_{0 h} e_{2 h} e_{1 h}^{2}\right)+\ldots\right]$

Neglecting the terms of $e_{h}{ }^{\prime} s$ having power greater than two we have

$$
\begin{aligned}
& \widehat{T}_{R P O}^{(S)}=\bar{Y}_{s t}\left[1+e_{0 h}-K_{h} e_{1 h}+K_{h}\left(e_{0 h} e_{1 h}+e_{1 h} e_{2 h}\right)+\frac{K_{h} \theta_{h}}{2} e_{1 h}^{2}\right] \text { or } \\
& \left(\widehat{T}_{R P O}^{(S)}-\bar{Y}_{s t}\right)=\sum_{h=1}^{L} W_{h} \bar{Y}_{h}\left[e_{0 h}-K_{h} e_{1 h}+K_{h}\left(e_{0 h} e_{1 h}+e_{1 h} e_{2 h}\right)+\frac{K_{h} \theta_{h}}{2} e_{1 h}^{2}\right]
\end{aligned}
$$

Now squaring both sides of (6.5) and neglecting the terms of $e_{h}{ }^{\prime} s$ having power greater than the second we have

$\left(\widehat{T}_{R P O}^{(S)}-\bar{Y}_{s t}\right)^{2}=\sum_{h=1}^{L} W_{h}^{2} \bar{Y}_{h}^{2}\left[e_{0 h}^{2}-K_{h}^{2} e_{0 h}^{2}-2 K_{h} e_{0 h} e_{1 h}\right]$

Taking the expectation of both sides of (6.6) we get the mean square error $M S E$ of $\widehat{T}_{R P O}^{(S)}$ to the first order of approximation as

$$
\begin{aligned}
& \operatorname{MSE}\left(\widehat{T}_{R P O}^{(S)}\right)=E\left(\widehat{T}_{R P O}^{(S)}-\bar{Y}_{s t}\right)^{2}=\sum_{h=1}^{L} W_{h}^{2} \bar{Y}_{h}^{2} E\left[e_{0 h}^{2}-K_{h}^{2} e_{0 h}^{2}-2 K_{h}\left(e_{0 h} e_{1 h}\right)\right] \\
& =\sum_{h=1}^{L} W_{h}^{2} \frac{\left(1-f_{h}\right)}{n_{h}} \bar{Y}_{h}^{2}\left[C_{y h}^{2}+K_{h}^{2} C_{x h}^{2}-2 K_{h} \rho_{h} C_{y h} C_{x h}\right] \\
& =\sum_{h=1}^{L} W_{h}^{2} \frac{\left(1-f_{h}\right)}{n_{h}} \bar{Y}_{h}^{2}\left[C_{y h}^{2}+\left(\rho_{h} \frac{C_{y h}}{C_{x h}}\right)^{2} C_{x h}^{2}-2\left(\rho_{h} \frac{C_{y h}}{C_{x h}}\right) \rho_{h} C_{y h} C_{x h}\right]
\end{aligned}
$$


Bajopas Volume 10 Number 1 June, 2017

$$
\begin{aligned}
& =\sum_{h=1}^{L} W_{h}^{2} \frac{\left(1-f_{h}\right)}{n_{h}} \bar{Y}_{h}^{2}\left(C_{y h}^{2}-\rho_{h}^{2} C_{y h}^{2}\right) \\
& =\sum_{h=1}^{L} W_{h}^{2} \frac{\left(1-f_{h}\right)}{n_{h}} \bar{Y}_{h}^{2} C_{y h}^{2}\left(1-\rho_{h}^{2}\right) \\
& =\sum_{h=1}^{L} W_{h}^{2} \frac{\left(1-f_{h}\right)}{n_{h}} S_{y h}^{2}\left(1-\rho_{h}^{2}\right)
\end{aligned}
$$

Which is equal to the minimum $M S E$ of $T_{R P}^{(S)}$ or the $M S E$ of $\hat{T}_{R P O}^{(S)}$ given by (3.10). Thus, we established that the $M S E$ of the estimator $\widehat{T}_{R P O}^{(S)}$ in (6.3), based on 'estimated optimum value' to the first degree of approximation is same as that of $\widehat{T}_{R P O}^{(S)}$ given by (3.9). So it is interesting to note the estimator $\widehat{T}_{R P O}^{(S)}$ in (6.3) can be used as an alternative to the $\widehat{T}_{R P O}^{(S)}$ given by (3.9) if the value of the parameter is not known.

Data Presentation for Stratified Random Sampling we consider a natural population data earlier considered by Singh and Chaudary (1986)given in page 162. $Y$ : Total number of Trees, $X$ : Area under orchards in hectares.

Table 7.1: Statistics of the Dataset

\begin{tabular}{cclll}
\hline Total & Stratum $\rightarrow$ & $\mathbf{1}$ & $\mathbf{2}$ & $\mathbf{3}$ \\
\hline$N=25$ & $N_{h}$ & 6 & 8 & 11 \\
$n=10$ & $n_{h}$ & 3 & 3 & 4 \\
$\bar{X}=8.379$ & $\bar{X}_{h}$ & 6.813 & 10.12 & 7.967 \\
$\bar{Y}=401.840$ & $\bar{Y}_{h}$ & 417.33 & 503.375 & 340.00 \\
$S_{x}^{2}=59.737$ & $S_{x h}^{2}$ & 15.97 & 132.66 & 38.438 \\
$S_{y}^{2}=12377.1$ & $S_{y h}^{2}$ & 74775.467 & 259113.70 & 65885.60 \\
$S_{x y}=2524.8$ & $S_{x y h}$ & 1007.055 & 5709.1629 & 1404.71 \\
$\rho_{x y}=0.9285$ & $\rho_{x y h}$ & 0.92152 & 0.9738 & 0.8827 \\
$R=49.031$ & $\gamma_{h}$ & 0.16667 & 0.2083 & 0.15909 \\
$\rho^{+}=0.941$ & $W_{h}^{2}$ & 0.0576 & 0.1024 & 0.1936 \\
$\mathbf{M}_{d}=4.349$ & $\mathrm{M}_{d}$ & 4.34932 & 4.34932 & 4.34932 \\
\hline
\end{tabular}

The merit of the proposed estimator $T_{R P}^{(S)}$ is illustrated using a real-life dataset. We compare the efficiency of the proposed estimator $T_{R P}^{(S)}$ with some other ratio and product estimators, i.e. $\bar{y}_{s t}, T_{R s t 1}, T_{P s t 1,} T_{R s t 2}$ and $T_{P s t 2}$ in Table 7.2 and Table 7.3

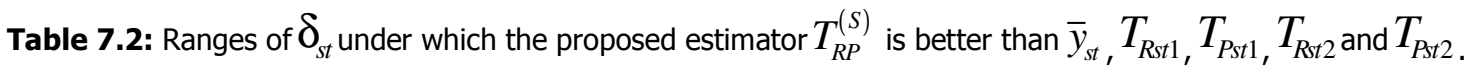

\begin{tabular}{lcc}
\hline Estimators & 1 & \\
\hline $\bar{y}_{s t}$ & $0.5<\delta_{s t}<1.933$ \\
$T_{R s t 2}$ & $1.0<\delta_{s t}<1.433$ \\
$T_{P s t 2}$ & $0.0<\delta_{s t}<2.433$ \\
$T_{R s t 1}$ & $1.138<\delta_{s t}<1.295$ \\
$T_{P s t 1}$ & $-0.295<\delta_{s t}<2.728$ \\
$\delta_{s t}$ & 1.2164 \\
\hline
\end{tabular}

\section{Percentage Relative Efficiency (PRE)}

We compute the percent relative efficiency of $\bar{y}_{s t}, T_{R s t 1}, T_{P s t 1}, T_{R s t 2}, T_{P s t 2}$ and the proposed estimator $T_{R P}^{(S)}$ with respect to $\bar{y}_{s t}$. The Percentage relative efficiency (PRE) of different estimators $T$ respect to $\bar{y}_{s t}$ is defined as, $P R E\left(T ., \bar{y}_{s t}\right)=\frac{V\left(\bar{y}_{s t}\right)}{V(T .)} \times 100$ 
Bajopas Volume 10 Number 1 June, 2017

Table 7.3: Percentage relative efficiency of various estimators with respect to $\bar{y}_{s t}$

\begin{tabular}{lll}
\hline Estimator & Variance & $\operatorname{PRE}\left(T ., \bar{y}_{s t}\right)$ \\
\hline $\bar{y}_{s t}$ & 8274.879 & 100 \\
$T_{R s t 1}$ & 1014.8035 & 815.42 \\
$T_{P s t 1}$ & 34998.554 & 23.64 \\
$T_{R s t 2}$ & 1437.01685 & 575.84 \\
$T_{P s t 2}$ & $21,538.7342$ & 38.42 \\
$T_{R P}^{(S)}$ & 842.5991 & 982.07 \\
\hline
\end{tabular}

\section{DISCUSSION}

We have proposed an estimator of the separate ratioproduct estimator and obtained the asymptotically optimum estimator (AOE) with its approximate MSE formula for the proposed estimator using the coefficient of variation and median of the auxiliary variable $X$ in stratified random sampling.

Theoretically, we have demonstrated that the proposed estimator is always more efficient than other estimators $\bar{y}_{s t}, T_{R s t 1}, T_{P s t 1}, T_{R s t 2}$ and $T_{P s t 2}$ under the effective ranges of $\delta_{s t}$ and its optimum values.

In addition, we support these theoretical results numerically using the data sets as shown in Table 7.1.

Table 7.2 provides us with the wide ranges of $\delta_{s t}$ along with its optimum values for which the proposed estimator $T_{R P}^{(S)}$ is more efficient than other estimators $\bar{y}_{s t}, T_{R s t 1}, T_{P s t 1}, T_{R s t 2}$ and $T_{P s t 2}$, as far as mean squared error criterion is considered. It is also observed from Table 4.6 that there is a scope for chosen $\delta_{s t}$ to obtain better estimators than $\bar{y}_{s t}, T_{R s t 1 \text {, }}$ $T_{P s t 1}, T_{R s t 2}$ and $T_{P s t 2}$

Table 7.3 provides that there is a considerable gain in efficiency by using proposed estimator $T_{R P}^{(S)}$ over the estimators $\bar{y}_{s t}, T_{R s t 1}, T_{P s t 1}, T_{R s t 2}$ and $T_{P s t 2}$. This shows that even if the scalar $\delta_{s t}$ deviates from with its optimum values $\left(\delta_{\text {opt. }}\right)$. The suggested estimator $T_{R P}^{(S)}$ will yield better estimate than $\bar{y}_{s t}, T_{R s t 1}, T_{P s t 1}$, $T_{R s t 2}$ and $T_{P s t 2}$.

$$
\begin{aligned}
& \operatorname{MSE}\left(\bar{y}_{s t}\right)-\operatorname{MSE}\left(T_{R P}^{(S)}\right)=\sum_{h=1}^{L} W_{h}^{2} \frac{\left(1-f_{h}\right)}{n_{h}} \bar{Y}_{h}^{2} C_{y h}^{2}>0 \\
& \operatorname{MSE}\left(T_{R s t 1}\right)-\operatorname{MSE}\left(T_{R P}^{(S)}\right)=\sum_{h=1}^{L} W_{h}^{2} \frac{\left(1-f_{h}\right)}{n_{h}} \bar{Y}_{h}^{2} C_{y h}^{2}\left(1-K_{h}\right)^{2}>0 \\
& \operatorname{MSE}\left(T_{P s t 1}\right)-\operatorname{MSE}\left(T_{R P}^{(S)}\right)=\sum_{h=1}^{L} W_{h}^{2} \frac{\left(1-f_{h}\right)}{n_{h}} \bar{Y}_{h}^{2} C_{y h}^{2}\left(1+K_{h}\right)^{2}>0 \\
& \operatorname{MSE}\left(T_{R s t 2}\right)-\operatorname{MSE}\left(T_{R P}^{(S)}\right)=\sum_{h=1}^{L} W_{h}^{2} \frac{\left(1-f_{h}\right)}{n_{h}} \bar{Y}_{h}^{2} C_{y h}^{2}\left(\theta_{h}-K_{h}\right)^{2}>0 \\
& \operatorname{MSE}\left(T_{P s t 2}\right)-\operatorname{MSE}\left(T_{R P}^{(S)}\right)=\sum_{h=1}^{L} W_{h}^{2} \frac{\left(1-f_{h}\right)}{n_{h}} \bar{Y}_{h}^{2} C_{y h}^{2}\left(\theta_{h}+K_{h}\right)^{2}>0
\end{aligned}
$$

It was observed from the analysis that in stratified random sampling that the proposed estimator have the minimum MSE and bias compared to some ratio and product estimators in existence and mean per unit estimator, and the proposed estimator attain its minimum MSE at its optimum value.

Evidence from the study revealed that the proposed estimator is more efficient than the already existing ratio, product and ratio- product type estimators based on some certain conditions and efficiency conditions. Therefore, there is always need to ensure that the auxiliary variable is highly correlated with the study variable. Also where there is correlation between the auxiliary variable and the study variable and such population is non-homogeneous, stratified random sampling will be more appropriate. when there is no correlation between the auxiliary variable and the study variable, the application of singlephase sample will not yield more efficient or the mean per unit will be more efficient. Hence, we conclude that the proposed class of estimator $T_{R P}^{(S)}$ is more efficient than the other estimators in terms of its optimality. Thus, it is preferred to use the proposed estimator $T_{R P}^{(S)}$ over $\bar{y}_{s t}, T_{R s t 1}, T_{P s t 1}, T_{R s t 2}$ and $T_{P s t 2}$ estimators in stratified random sampling. 


\section{REFERENCES}

Cochran, W.G. (1940). The estimation of the yield of the cereal experiments by sampling for ratio of grain to total produce. The journal of Agricultural Science,30: 262-275.

Das, A.K. and Tripathi, T.P. (1981). A class of sampling strategies for population mean using information on mean and variance of an auxiliary character. Proceedings of Italian Statistical Institute, Golden Jubilee International Conference of Statistics. Applications and new directions,174-181.

Housila, P.S. and Neha-Agnihotri (2008). A general procedure of estimating population mean using auxiliary information in sample surveys. Statistics in Transition-new series, 9(1):71-87.

Kadilar, C. and Cingi, H. (2003). Ratio estimators in stratified random sampling. Biometrical Journal, 2:218-225.

Okafor, F. C. (2002). Sample Survey Theory and Applications ( $1^{\text {st }}$ edition). Nsukka, Nigeria.

Perri, P.F. (2005). Combination of auxiliary variables in ratio-cum-product type estimators. In: Proceedings of Italian Statistical Society, Intermediate meeting on Statistics and Environment, Messina, Italy, September 2005,pp. 193-196.

Reddy, V.N. and Singh, H.P. (1978). A study on the use of prior knowledge on certain population parameters in estimation, Sankhya C, 40: 2937.
Singh, H.P and Tailor, R. (2003). Estimation of finite population mean using known correlation coefficient of auxiliary characters. Statistica, Anno LXV, 4:407-418.

Singh, H.P and Tailor, R. (2005). Estimation of finite population mean with known coefficient of variation of auxiliary characteristic. Statistica, anno LXV, (3):301-313.

Singh, D. and Chaudary, F.S. (1986). Theory and analysis of sample survey Designs. New Age International Publisher.pp. 177-178.

Singh, H.P. and Kakaran, M.S. (1993). A modified ratio estimator using known coefficient of kurtosis of an auxiliary character. Journal of Indian Society of Agricultural Statistics, New Delhi, India.

Singh, H.P. and Ruiz Espejo, M. (2003). On linear regression and ratio-product estimation of a finite population mean. The Statistician, 52(1): 59-67.

Singh, H.P. and Vishwakarma, G.K. (2011). Seperate ratio- product estimator for estimating population mean using auxiliary information. Journal of Statistical Theory and Application. 10(4): 653-664.

Subramani, J. and Kumarapandiyan, G. (2012). Estimation of population mean using coefficient of variation and median of the auxiliary variable. International Journal of Probability Statistics, 1(4): 111-118, DOI 10.5923/j. ijps.20120104.04. 